\title{
Officers King and Zeng and the Case of the Unsupported Counterfactual
}

\author{
JAMES D. MORROW \\ University of Michigan
}

Let me begin by praising Gary King and Langche Zeng for their service to the field with the methods they describe in their paper (King and Zeng 2006a). It is important to know when counterfactual statements drawn from statistical estimates wander far from the data used in the estimates. I particularly like the subtlety of the observation that even when all the values of the individual variables occur in the data, it may not be the case that all combinations of those values do. There are countries that protect the civil liberties of their citizens and those that abuse them and there are democracies and autocracies, but there are not very many autocracies that protect civil liberties or democracies that abuse them. Consequently, our inferences about autocracies with civil liberties and democracies without them are not based on the same depth of data that our inferences about democracies with civil liberties and autocracies without them are. I am looking forward to using their methods to examine some of my own recent data analysis on compliance with the laws of war. ${ }^{1}$ For example, U.S. military dominance characterized the Kosovo bombing and the Iraq War, but how different were these cases from the record of earlier twentieth century wars?

Like Shakespeare's Marc Anthony, however, one does not get asked to contribute to such fora to praise Caesar but to bury him. While I have neither the desire, the ability, nor the intent to bury Professors King and Zeng, I do have a clod or two of dirt to toss in their general direction. My qualifications to their excellent and important article concern theory as the source of counterfactual conclusions and the range of evidence we can use to judge among competing theories.

Their paper seems to assume that theory is fully captured by a well-specified regression equation, including functional form of the relationship, as judged by its ability to represent a given data set. It is as if they draw their epistemological inspiration from two fictional police officers: Captain Louis Renault of the movie "Casablanca," "Round up the usual suspects!," and Sergeant Joe Friday from the TV series "Dragnet," "Just the facts, ma'am." At times, I get the impression that theory plays no role in their process beyond providing a list of exogenous variables - the usual suspects - along with the appropriate specification of functional form and link function between those variables and the dependent variable, which is then applied to just the facts. But theories are more than just a list of relevant variables and a functional form; they are logical structures that provide arguments about why the variables cause one another. There are other forms of investigation than those of Captain Renault and Sergeant Friday.

Theory is critical for counterfactuals because theories explain why the counterfactual should hold. Reasons why the counterfactual should be true are as

\footnotetext{
Author's note: I would like to thank Orit Kedar for her comments on this response.

${ }^{1}$ See Morrow and Jo (2006) for a description of this data and some simple analysis.
} 
important as the evidentiary base behind a counterfactual inference. ${ }^{2}$ When I was in college, I had a friend who calculated what the universe would look like if the speed of light was 30 miles per hour. He used the theory of general relativity he learned in class and made the calculations of the theory after changing the speed of light. He did this to see what it would be like if he could drive his old MG-B sports car at near light speed. This counterfactual has no empirical basis whatsoever and would fail King and Zeng's tests spectacularly. My friend's counterfactual universe had credibility because general relativity has been tested sufficiently that it is widely accepted in physics. Empirical work helps us as a scientific community to determine which theories represent the world best and so form the best basis for counterfactual inference. Of course, we may find a well-established theory replaced because it fails to represent elements of reality that we have not fully investigated and so leads to counterfactuals that prove incorrect upon observation.

We test theories by deducing conclusions from them and comparing those conclusions to reality, where such comparisons could be done through a large- $n$ statistical test or the close examination of individual cases. In some cases, the evidence we have may not be sufficient to judge between competing theories; King and Zeng provide an example where the available data does not distinguish between a linear and a quadratic relationship because it lacks sufficient variance on the independent variable in question. One way to try and separate theories is to derive novel hypotheses from them which separate the two theories. The evidence then may be decisive in judging between the two and allow us to make counterfactual inferences with shared confidence. Here King and Zeng's Sergeant Friday-like focus on just the facts could lead the reader away from developing the logic of the theories in question to test them against novel hypotheses that might separate them. Their methods could help us identify situations where the existing data does not separate competing theories and so direct us toward the derivation and testing of additional hypotheses.

This omission is particularly important for international studies because many scholars of international studies use case studies precisely to test theoretically derived hypotheses which may not be amenable to a large- $n$ quantitative test. Some variables may not be easily collected for a large number of cases; this is one of the motivations King and Zeng (2001) give for the use of their rare events logit technique. More broadly, case studies might be helpful in studying causal explanations because they allow us to test more hypotheses drawn from competing explanations (see King, Keohane, and Verba 1994:29-31, 43-46, 217-28). Again, we could have greater confidence in our counterfactual claims because we would have greater confidence about which theory was better than could be provided simply by a large- $n$ regression equation.

King and Zeng's methods could also be useful if we believe that the combinations of variables that we do not find in our data are combinations that cannot occur, rendering the counterfactual meaningless. Returning to my earlier example, there are few cases of autocracies with full protection of civil liberties. Whether Mikhail Gorbachev's experiment with glasnost under communism would lead to economic growth or other desirable outcomes then was an unsupportable counterfactual. It is helpful to know when we are making an implausible counterfactual because the combination of values in that counterfactual does not occur. Again however, confidence in such an argument requires more than just a demonstration that a

\footnotetext{
${ }^{2}$ Tetlock and Belkin (1996:16-31) provide six criteria for judging counterfactuals: well-specified antecedents and consequents, logical consistency of connecting principles, consistency with well-established historical facts, consistency with well-established theoretical laws, consistency with well-established statistical generalizations, and projectability. King and Zeng's method only addresses the fifth of these criteria. See Dawes (1996) for a spirited defense of a position close to that of King and Zeng's method, that counterfactuals necessarily rely on valid statistical generalizations.
} 
particular combination of values does not occur in the data; it requires a theory that explains why this combination of values does not occur. Continuing the example from my opening paragraph, Bueno de Mesquita, Smith, Siverson, and Morrow (2003:180-82) provide an explanation of why democracy and civil liberties are necessarily found together. Democratic leaders must hold the loyalty of a large winning coalition, which is done more effectively with public goods, such as civil liberties, than with private benefits. Obviously, this is not the only explanation of this pattern, but it illustrates the point that ruling out the counterfactual of autocracies with civil liberties depends not just on the lack of such cases in the data but on a theory that explains why we should not observe cases with that combination of the variables.

Let me end my brief comment by returning to my appreciation for the value of the methods that King and Zeng describe in their paper. I have argued that there are ways forward in judging counterfactuals even when the evidence used in a large- $n$ study lacks the variability to provide a full evidentiary base for those counterfactuals. Theories make counterfactual predictions, and we have ways to help us judge among competing theories that may not be separable with existing data. Still, their methods for judging whether a given counterfactual falls far from the data we have can help us see when that is true. To be fair, there are many theories used in international studies that fail to meet the standards that I prescribe; some are little more than loose verbal arguments that are consistent with many regression specifications. Such theories may not support the logical derivation of novel hypotheses necessary for careful testing when the evidence does not allow for clearly supported counterfactuals. King and Zeng's methods will help us understand the limits of our data analysis in such cases. I come back to my appreciation for their contribution because such fora focus on our differences rather than our agreements, to toss clods of dirt instead of praise. I hope my gentle admonition to Professors King and Zeng to move beyond the methodological prescriptions of Captain Renault and Sergeant Friday will not lead them to adopt the methods of a third fictional police officer, Inspector "Dirty" Harry Callahan, in their response: “... you've got to ask yourself one question: 'Do I feel lucky?' Well, do ya, punk?”

\section{References}

Bueno de Mesquita, Bruce, Alastair Smith, Randolph M. Siverson, and James D. Morrow. (2003) The Logic of Political Survival. Cambridge, MA: The MIT Press.

DAwEs, Robyn M. (1996) Counterfactual Inferences as Instances of Statistical Inferences. In Counterfactual Thought Experiments in World Politics, edited by Philip E. Tetlock and Aaron Belkin. Princeton, NJ: Princeton University Press.

King, Gary, Robert O. Keohane, and Sidney Verba. (1994) Designing Social Inquiry: Scientific Inference in Qualitative Research. Princeton, NJ: Princeton University Press.

King, GARY, AND LANGChe Zeng. (2001) Explaining Rare Events in International Relations. International Organization 55:693-716.

King, Gary, and Langche Zeng. (2007) When Can History Be Our Guide? The Pitfalls of Counterfactual Inference. International Studies Quarterly, this issue.

Morrow, James D., and Hyeran Jo. (2006) Compliance with the Laws of War: Dataset and Coding Rules. Conflict Management and Peace Science 23:91-113.

Tetlock, Philip E., And Aaron Belkin. (1996) Counterfactual Thought Experiments in World Politics: Logical, Methodological, and Psychological Perspectives. In Counterfactual Thought Experiments in World Politics, edited by Philip E. Tetlock and Aaron Belkin. Princeton, NJ: Princeton University Press. 
